\title{
Abnormal postnatal maintenance of elevated DLK1 transcript levels in callipyge sheep
}

\author{
Susan K. Murphy, ${ }^{1}$ Brad A. Freking, ${ }^{2}$ Timothy P.L. Smith ${ }^{2}{ }^{2}$ Kreg Leymaster, ${ }^{2}$ \\ Catherine M. Nolan, ${ }^{3}$ Andrew A. Wylie, ${ }^{1}$ Heather K. Evans, ${ }^{1}$ Randy L. Jirtle ${ }^{1}$ \\ ${ }^{1}$ Department of Radiation Oncology, Duke University, Box 3433, Durham, North Carolina 27710, USA \\ ${ }^{2}$ Agricultural Research Service, U.S. Meat Animal Research Center, U.S. Department of Agriculture, Clay Center, \\ Nebraska 68933-0166, USA \\ ${ }^{3}$ Zoology Department, University College Dublin, Belfield, Dublin 4, Ireland
}

Received: 28 June 2004 / Accepted: 2 December 2004

\begin{abstract}
The underlying mechanism of the callipyge muscular hypertrophy phenotype in sheep (Ovis aries) is not presently understood. This phenotype, characterized by increased glycolytic type II muscle proportion and cell size accompanied by decreased adiposity, is not visibly detectable until approximately three to eight weeks after birth. The muscular hypertrophy results from a single nucleotide change located at the telomeric end of ovine Chromosome 18, in the region between the imprinted MATERNALLY EXPRESSED GENE 3 (MEG3) and DELTA, DROSOPHILA, HOMOLOGLIKE 1 (DLK1) genes. The callipyge phenotype is evident only when the mutation is paternally inherited by a heterozygous individual. We have examined the pre- and postnatal expression of MEG3 and $D L K 1$ in sheep of all four possible genotypes in affected and unaffected muscles as well as in liver. Here we show that the callipyge phenotype correlates with abnormally high $D L K 1$ expression during the postnatal period in the affected sheep and that this elevation is specific to the hypertrophy-responsive fast-twitch muscles. These results are the first to show anomalous gene expression that coincides with both the temporal and spatial distribution of the callipyge phenotype. They suggest that the effect of the callipyge mutation is to interfere with the
\end{abstract}

\footnotetext{
Mention of a trade name, proprietary product, or specified equipment does not constitute a guarantee or warranty by the USDA and does not imply approval to the exclusion of other products that may be suitable.

Correspondence to: Randy L. Jirtle; E-mail: jirtle@radonc. duke.edu
}

normal postnatal downregulation of DLK1 expression.

Callipyge (Greek for "beautiful buttocks") was the name given to the phenotype observed in certain descendants of a ram named "Solid Gold," born to a sheep (Ovis aries) flock in Oklahoma in 1983. Callipyge sheep exhibit muscular hypertrophy characterized by an elevation in number and size of type IIb glycolytic (fast-twitch) muscle fibers (Koohmaraie et al. 1995), including those in the longissimus dorsi and biceps femoris muscles. This is not a global muscle attribute, in that other muscles within the affected sheep (e.g., infraspinatus) are not subject to hypertrophy. The callipyge phenotype is inherited in a manner that is similar to that observed for imprinted genes; however, callipyge is apparent only in heterozygotes in which the mutation is inherited from the sire $\left(N^{M A T} C^{P A T}\right.$, where $N$ indicates the wild-type allele, $C$ denotes the mutant allele, and the superscripts MAT and PAT indicate maternally and paternally derived, respectively). Unexpectedly, sheep homozygous for the mutation $\left(C^{M A T} C^{P A T}\right)$ are phenotypically normal despite paternal inheritance of the mutated allele. This mode of inheritance was given the designation "polar overdominance" (Cockett et al. 1996) because the alternate heterozygotes exhibit divergent phenotypes (polar) and both homozygote genotypes are phenotypically normal (overdominance). Although highly suggestive of an imprinting component, the incomplete penetrance in the homozygous $C^{M A T} C^{P A T}$ individuals is not consistent with a phenotype strictly mediated by altered imprinting. 


\section{Ovis aries}

\section{Chromosome 18}

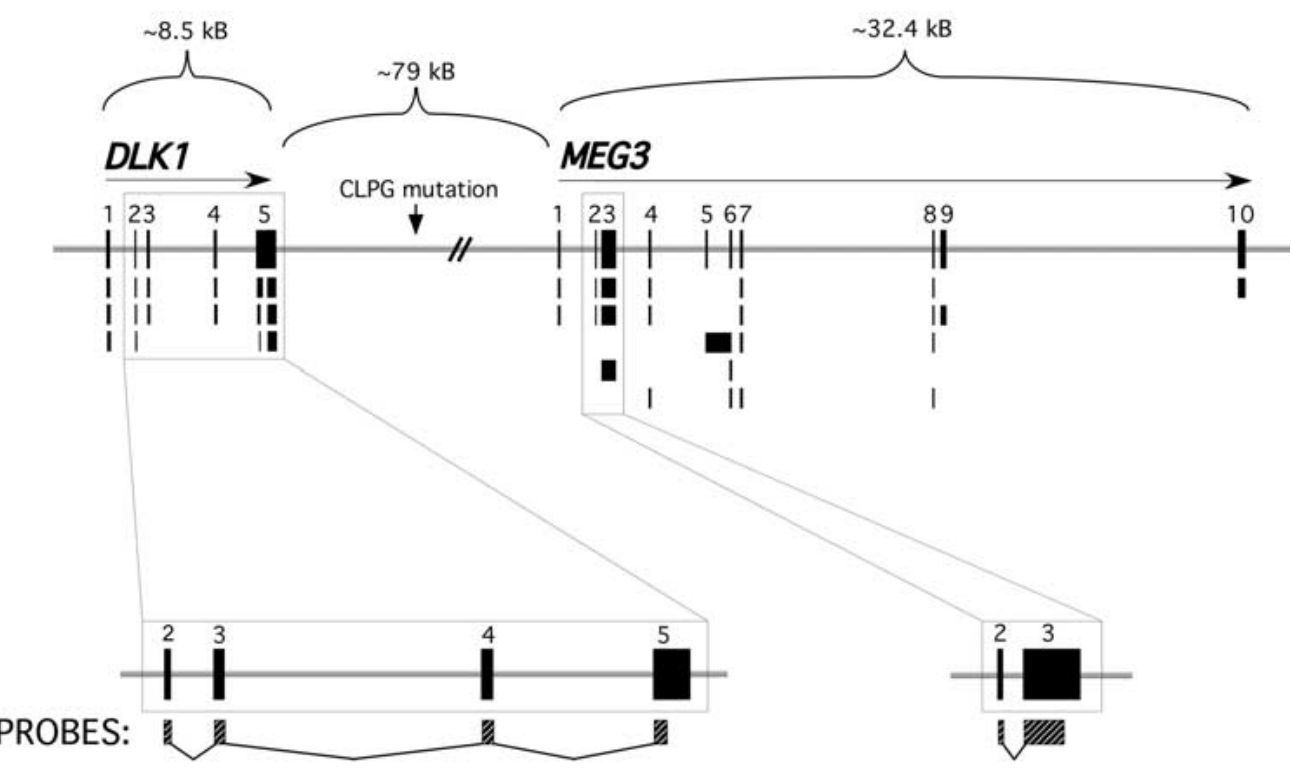

DLK1: 373 bases protected

MEG3: 294 bases protected

Fig. 1. Schematic representation of the DLK1/MEG3 locus on ovine Chromosome 18. The direction of transcription is indicated by the arrows. Paternally expressed $D L K 1$ and maternally expressed MEG3 are separated by approximately $79 \mathrm{~kb}$ and the relative position of the causative callipyge mutation is shown. The position of riboprobes designed to detect $D L K 1$ and MEG3 [variant A (Bidwell et al. 2001)] transcripts is indicated below. Exon numbering for DLK1 is based on information for the bovine (Fahrenkrug et al. 1999) and ovine (Charlier et al. 2001b) DLK1 genomic structure. MEG3 exons represent all known splice variants (Bidwell et al. 2001).

The nearly 10-year search for the mutation that causes the callipyge phenotype in sheep recently concluded with the identification of a single nucleotide change located within an intergenic region of the DLK1 (DELTA, DROSOPHILA, HOMOLOGLIKE 1) and MEG3 (MATERNALLY EXPRESSED GENE 3, also referred to as GTL2) imprinted domain (Fig. 1) (Freking et al. 2002; Smit et al. 2003). In spite of the identification of this mutation, two things remain unclear: first, the molecular mechanism that allows a single nucleotide change in an apparently intergenic region to give rise to callipyge and, second, why the homozygous callipyge animals are seemingly unaffected.

There are several other imprinted genes in addition to DLK1 and MEG3 in the vicinity of the callipyge mutation. Charlier et al. (2001a) reported that the imprint status of these genes, including $D A T$ (DLK1-Associated Transcript), PEG11 (Paternally Expressed Gene 11), MEG8 (Maternally Expressed Gene 8), and the aforementioned genes DLK1 and $M E G 3$ was not altered in any of the callipyge genotypes evaluated. We independently confirmed the monoallelic expression of these genes in both fetal and adult sheep (unpublished data). The callipyge mutation therefore does not alter the imprinting per se of nearby genes; this suggests instead that other aspects of the regulated expression of these genes may be affected.

The muscular hypertrophy of $N^{M A T} C^{P A T}$ animals occurs only in fast-twitch muscles and does not become apparent until after birth, indicating temporal regulation of the causative gene. We therefore predicted that the altered expression pattern of such a gene or genes would (1) be specific to sheep demonstrating the callipyge phenotype $\left(N^{M A T} C^{P A T}\right.$ sheep) and not be observed in animals with other genotypes, (2) be specific to muscles affected by the mutation, and (3) be concurrent with the appearance of the phenotype.

The known biological activities of DLK1 strongly suggest that this protein contributes to the etiology of the callipyge phenotype. DLK1 is similar to the Drosophila neurogenic protein Delta (Laborda et al. 1993) and belongs to the epidermal growth factor-like family of proteins. DLK1 functions in regulating adipogenesis and cellular growth and differentiation (Laborda et al. 1993; Laborda 2000), including neu- 
roblast differentiation (Van Limpt et al. 2000, 2003). Dlk1 knockout mice exhibit growth retardation, rapidly develop obesity, and are unable to maintain appropriate levels of lipid metabolites, demonstrating important antiadipogenic and growth roles for murine Dlkl (Moon et al. 2002). DLK1 is indeed highly expressed in preadipocytes and inhibits their differentiation, and is suppressed once the differentiation process begins (Smas and Sul 1993). Carcasses from callipyge sheep are characterized by decreased adiposity with approximately $24.3 \%$ fat and $71.3 \%$ fat-free lean compared with $31.5 \%$ and $64.0 \%$ for normal carcasses at typical industry slaughter weights (Freking et al. 1998b). The close proximity of sheep DLK1 to the callipyge mutation, together with the roles of DLK1 in growth and differentiation processes, led us to previously propose that this gene is an excellent candidate for involvement in development of the callipyge phenotype (Fahrenkrug et al. 1999). Based on the known functions of DLK1, altered expression due to paternal inheritance of the callipyge mutation may be responsible for the observed lean phenotype of the callipyge sheep.

As an initial step in studying gene expression that may be affected by the callipyge mutation, we chose to examine DLK1 and MEG3. MEG3 encodes RNA that lacks a significant open reading frame and whose function remains unknown. There are multiple alternatively spliced transcripts of $M E G 3$ in mice (Croteau et al. 2003), humans (UCSC Genome Browser, July 2003; http://genome.ucsc.edu//, and sheep (Bidwell et al. 2001) without preservation of potential coding sequence, suggesting that MEG3 functions as a noncoding RNA. DLK1 and MEG3 may exhibit coregulated expression, since they share many features of the coordinately regulated IGF2/ H19 imprinted gene pair that also influence growth and development (Wylie et al. 2000; Paulsen et al. 2001; Takada et al. 2002). Furthermore, MEG3 has been postulated to regulate $D L K 1$ expression in trans (Georges et al. 2003), as has $H 19$ also been implicated as a trans regulator of IGF2 (Forne et al. 1997; Li et al. 1998; Runge et al. 2000). We therefore examined the pre- and postnatal profile of gene expression for MEG3 and DLK1 to determine whether the presence of the mutation contributes to deregulated transcription of these genes in a tissue-specific and/or ontogenic manner.

\section{Materials and Methods}

Tissues. Sheep muscle tissue (longissimus dorsi, biceps femoris, infraspinatus) and liver were obtained from a resource population produced and maintained at the United States Department of
Agriculture Meat Animal Research Center in Clay Center, Nebraska (Freking et al. 1998a). Tissues were stored at $-80^{\circ} \mathrm{C}$.

Nucleic acid purification. Total RNA was isolated from frozen tissues using RNA Stat-60 according to the manufacturer's directions (TelTest), resuspended in nuclease free water, and either used immediately or stored at $-80^{\circ} \mathrm{C}$ prior to use.

Quantitative RNA analysis. $3.0 \mu \mathrm{g}$ of total RNA from fetal (97-133 days gestation; see Freking et al. 1998a) $\left[N^{M A T} N^{P A T} \quad(n=4), \quad C^{M A T} N^{P A T} \quad(n=3)\right.$, $N^{M A T} C^{P A T}(n=4)$, and $\left.C^{M A T} C^{P A T}(n=2)\right]$ and adult (233-240 days) sheep $\left[N^{M A T} N^{P A T}(n=2), C^{M A T} N^{P A T}\right.$ $(n=2), \quad N^{M A T} C^{P A T}(n=4)$, and $\left.C^{M A T} C^{P A T}(n=2)\right]$ was analyzed using RNase protection assays (RPAII kit; Ambion, Austin, TX). Probes for DLK1 and $M E G 3$ were prepared by PCR amplification using oligo dT-primed sheep cDNA with oligonucleotide primers for DLK1: DLKX2F (5'-GAA TGC TTC CCG GCC TGC CAC C-3') and DLKX5R (5' CCC TCA TCG TCC ACG CAG C-3') and for MEG3: MEG3F1 (5'-GAC ACC TTC CGT CTG CCT TCC-3') and MEG3R9 (5' CAG AAG CAG ACG CEAC ATA GAA AAG C-3'). The MEG3 primers were designed based on the known bovine sequence because the ovine sequence including MEG3 (Accession No. AF354168) was not available at the time these studies were initiated. The underlined residues in the MEG3 primer sequences represent mismatches between the ovine and the bovine sequence, but these did not hinder the ability to amplify the MEG3 sequence from ovine cDNA.

The amplicons were cloned into pGemT-Easy vectors (Promega) followed by nucleotide sequence confirmation from insert-containing colonies. The 373-nt DLK1 probe sequence (within the 448-nt total length probe) corresponds to positions 54,868-61,074 of accession No. AF354168, and the 311-nt MEG3 probe sequence /which protects a 294-nt fragment due to mismatches in the primer sequences within the 397-nt total length probe) corresponds to positions 140,792-141,315 of Accession No. AF354168. Both cDNA probes were designed to contain sequence from multiple exons. Antisense probes were generated by in vitro transcription (MaxiScript, Ambion) from linearized templates in the presence of $\alpha^{32} \mathrm{P}$-[rCTP] using the Ambion Maxiscript kit. 18S rRNA was used as an internal control with $\alpha^{32} \mathrm{P}$ [rCTP]-labeled probes generated from the pTRIRNA-18S vector using the MEGAscript kit (Ambion). The protected DLK1, MEG3, and 18S rRNA fragments were separated on $6 \%$ denaturing polyacrylamide gels and quantified using a Molecular 
Dynamics Storm PhosphorImager System (Amersham Biosciences, Piscataway, NJ). Relative levels of the protected DLK1 and MEG3 transcripts were normalized to the protected $18 \mathrm{~S}$ rRNA fragments present in each sample.

Statistical analysis of the expression levels for each gene was performed using the unpaired Student's $t$-test with a two-tailed distribution assuming heteroscedastic variance. Unless otherwise specified, comparisons were made relative to normal sheep. Probability values less than 0.05 were considered significant.

\section{Results}

We predicted that a callipyge effector gene(s) would exhibit altered patterns of expression among the callipyge genotypes that correlate with the presence and absence of the phenotype. To this end, we used RNase protection assays (Fig. 1) to measure expression of MEG3 and DLK1 in the hypertrophyresponsive longissimus dorsi and biceps femoris muscles, nonhypertrophic infraspinatus muscle, and liver from sheep of all four genotypes $\left(N^{M A T} N^{P A T}\right.$, $C^{M A T} N^{P A T}, N^{M A T} C^{P A T}$, and $\left.C^{M A T} C^{P A T}\right)$.

During prenatal development, we found low levels of MEG3 transcripts in lonsissimus dorsi muscle of $N^{M A T} N^{P A T}$ and $N^{M A T} C^{P A T}$ animals, with a modest increase in fetuses with maternal inheritance of the callipyge mutation (Fig. 2A). This trend is maintained in the adult sheep, with an overall elevation in $M E G 3$ transcript levels relative to those in the fetal sheep of the same genotype (Fig. 2A). The increased level of $M E G 3$ mRNA observed in the fetal $C^{M A T} N^{P A T}$ and $C^{M A T} C^{P A T}$ sheep is even more prominent in the adults, but these differences were not statistically significant.

To determine the expression levels of $D L K 1$, we measured DLK1 transcripts in fetal and adult longissimus dorsi. DLK1 expression is high prenatally in all four genotypes with the maximum level of expression in the $C^{M A T} N^{P A T}$ animals (Fig. 3A, 3B). Postnatally, there is an approximate threefold decrease in the expression of DLK1 in the $N^{M A T} N^{P A T}$, $C^{M A T} N^{P A T}$, and $C^{M A T} C^{P A T}$ sheep, but strikingly, the $N^{M A T} C^{P A T}$ sheep maintain elevated levels of DLK1 transcripts $(p=0.013$ compared to that of the $N^{M A T} N^{P A T}$ animals; Fig. 3A, 3C). Our results indicate that $N^{M A T} C^{P A T}$ sheep do not repress postnatal transcription of $D L K 1$.

To examine whether these patterns of expression are specific to muscles containing predominantly fast-twitch fibers (the muscles affected in the callipyge sheep), we evaluated MEG3 and DLK1 expression in two other muscle types in adult sheep. Biceps femoris, another hypertrophy-responsive muscle, exhibits a very similar profile of MEG3 expression to longissimus dorsi, with elevated MEG3 levels in sheep with a maternally inherited callipyge mutation (Fig. 4A). Interestingly, this pattern is also seen in infraspinatus (Fig. 4B), which is composed primarily of slow-twitch muscle fibers and is unaffected in the callipyge phenotype. Although the levels of infraspinatus MEG3 transcripts are higher in sheep inheriting the callipyge mutation on the maternal allele compared to normal sheep, these differences were not significant. Fetal and adult $N^{M A T} C^{P A T}$ sheep do not express significantly altered levels of MEG3 relative to $N^{M A T} N^{P A T}$ sheep in any of the hypertrophic muscle types analyzed. These results indicate that the influence of the callipyge mutation on expression of MEG3 is not restricted to fast-twitch muscle fibers and therefore suggest that $M E G 3$ expression is not directly responsible for the callipyge phenotype.

DLK1 expression is increased in biceps femoris of the $N^{M A T} C^{P A T}$ animals approximately fourfold over that observed in the $N^{M A T} N^{P A T}(p=0.042)$ and $C^{M A T} N^{P A T}$ animals $(p=0.039)$ (Fig. 4A). Biceps femoris in the $C^{M A T} C^{P A T}$ animals exhibits slightly higher levels of $D L K 1$ transcripts relative to the $N^{M A T} N^{P A T}$ and $C^{M A T} N^{P A T}$ sheep, but the expression is much lower $(\sim 40 \%)$ than in $N^{M A T} C^{P A T}$ sheep $(p=0.114)$. Although biceps femoris is an affected muscle in the callipyge sheep, our results indicate that the increase in DLK1 expression in this particular muscle is not as high as that observed in longissimus dorsi. However, DLK1 expression in biceps femoris in the $N^{M A T} C^{P A T}$ adult sheep is distinctly elevated relative to the other three genotypes.

In contrast to the elevated $D L K 1$ gene expression profile seen in adult $N^{M A T} C^{P A T}$ fast-twitch muscles, $D L K 1$ is expressed at much lower levels in infraspinatus of the same adult sheep (Fig. 4B). We observed small elevation in expression in the $C^{M A T} N^{P A T}$ (1.8-fold), $N^{M A T} C^{P A T}$ (1.6-fold), and $C^{M A T} C^{P A T}$ (1.7fold) adult animals relative to the $N^{M A T} N^{P A T}$ sheep. In spite of this small elevation in DLK1 levels across all three genotypes carrying the mutated allele(s), the differences in expression for both DLK1 and MEG3 between $N^{M A T} C^{P A T}$ and $N^{M A T} N^{P A T}$ adult sheep are statistically significant $(p=0.027$ and $p=0.031$, respectively). This finding is likely due to the greater precision of testing differences between a larger number of $N^{M A T} C^{P A T}$ individuals $(n=4)$ relative to the other genotypes ( $n=2$ for each), since $C^{M A T} N^{P A T}$ and $C^{M A T} C^{P A T}$ both express higher averaged levels of $D L K 1$ and $M E G 3$ than do the $N^{M A T} C^{P A T}$ animals. These results indicate that the abnormal elevation of $D L K 1$ expression in the adult $N^{M A T} C^{P A T}$ animals 


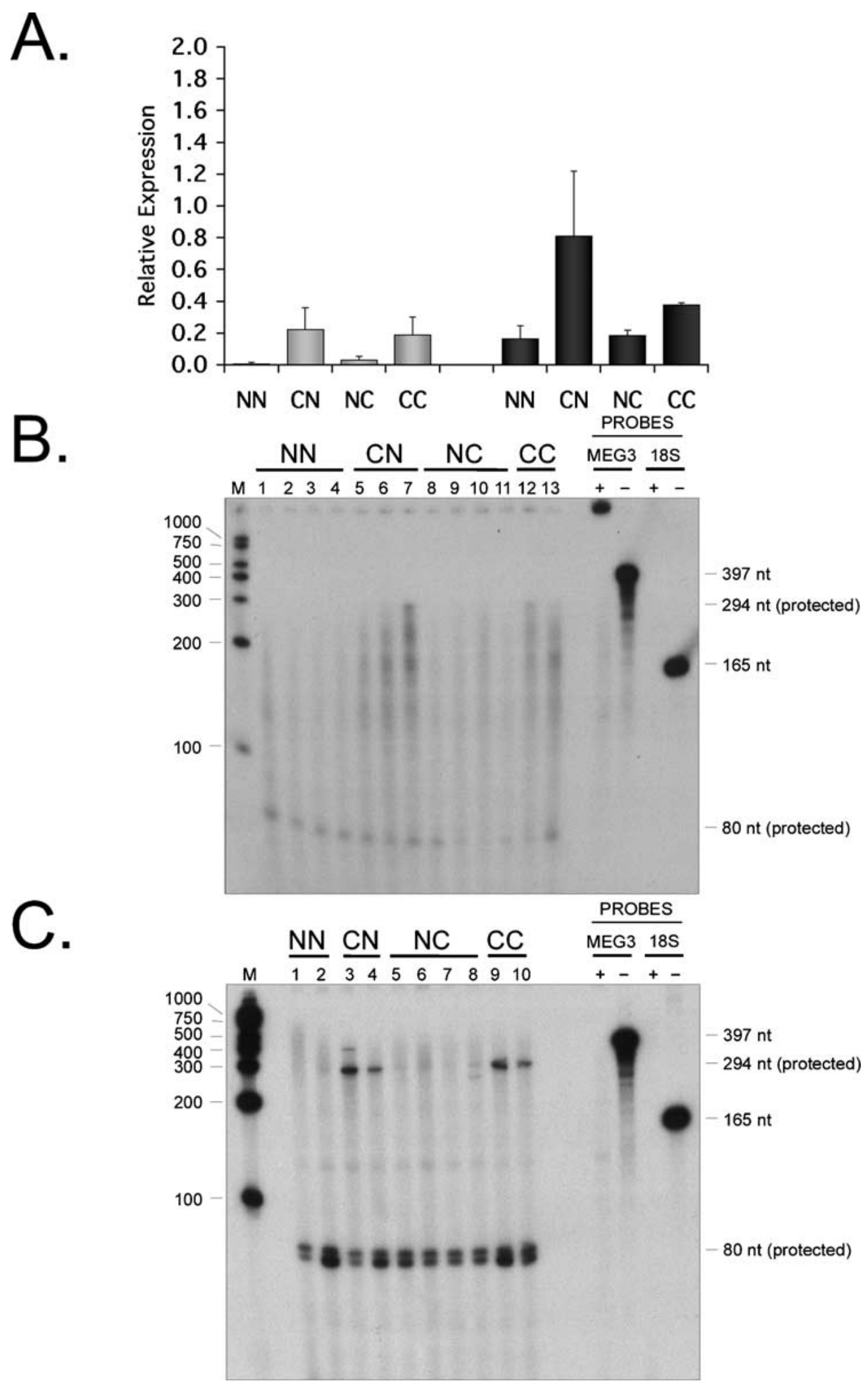

Fig. 2. Quantitative analysis of MEG3 expression in longissimus dorsi of fetal and adult sheep. Total RNA from longissimus dorsi muscle was analyzed using RNase protection and normalized to $18 \mathrm{~S}$ rRNA present in each sample to give the relative level of gene expression. (A) Relative expression of MEG3 in fetal longissimus dorsi (gray bars) from $N^{M A T} N^{P A T}(n=4), C^{M A T} N^{P A T}(n=3), N^{M A T} C^{P A T}(n=4)$, and $C^{M A T} C^{P A T}(n=2)$ sheep and adult longissimus dorsi (black bars) from $N^{M A T} N^{P A T}(n=2), C^{M A T} N^{P A T}(n=2), N^{M A T} C^{P A T}(n=4)$, and $C^{M A T} C^{P A T}(n=2)$ sheep. Error bars, standard error of the mean (SEM). (B, C) RNase protection assays used to generate the histogram in panel (A) showing expression of $M E G 3$ in fetal $(\mathbf{B})$ and adult $(\mathbf{C})$ longissimus dorsi. Probes were run in the presence $(+)$ or absence $(-)$ of RNase. The MEG3 probe protects 294 nucleotides of MEG3 variant A (Bidwell et al. 2001), while the 18 S rRNA probe protects a doublet 

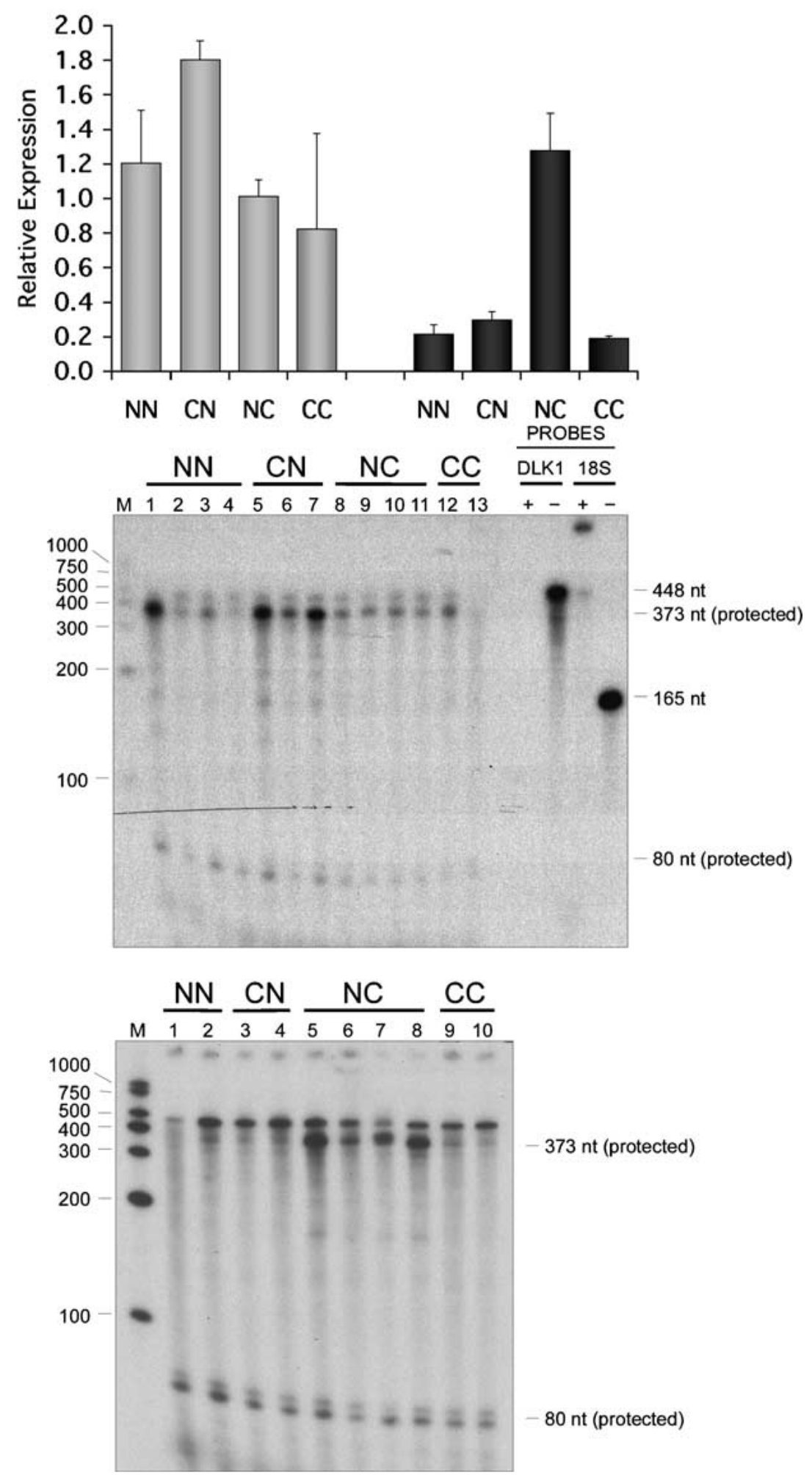

Fig. 3. Quantitative analysis of DLK1 expression in longissimus dorsi of fetal and adult sheep. Total RNA from longissimus dorsi muscle was analyzed using RNase protection and normalized to $18 \mathrm{~S}$ rRNA present in each sample to give the relative level of gene expression. (A) Relative expression of DLK1 in fetal longissimus dorsi (gray bars) from $N^{M A T} N^{P A T}(n=4), C^{M A T} N^{P A T}(n=3), N^{M A T} C^{P A T}(n=4)$, and $C^{M A T} C^{P A T}(n=2)$ sheep and adult longissimus dorsi (black bars) from $N^{M A T} N^{P A T}(n=2), C^{M A T} N^{P A T}(n=2), N^{M A T} C^{P A T}(n=4)$, and $C^{M A T} C^{P A T}(n=2)$ sheep. Error bars-SEM. (B, C) RNase protection assays used to generate the histogram in panel (A) showing expression of DLK1 in fetal (B) and adult (C) longissimus dorsi. Probes in $(\mathbf{B})$ were run alone in the presence $(+)$ or absence $(-)$ of RNase. The DLK1 probe protects 373 nucleotides, while the $18 \mathrm{~S}$ rRNA probe protects a doublet migrating at approximately 80 nucleotides. M-marker lane. 


\section{A. Biceps femoris}

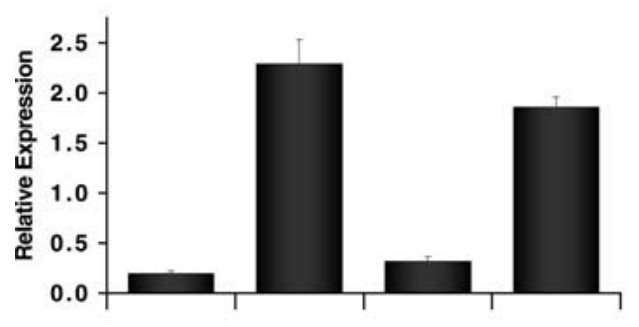

NN CN NC $\propto$

MEG3

\section{B. Infraspinatus}

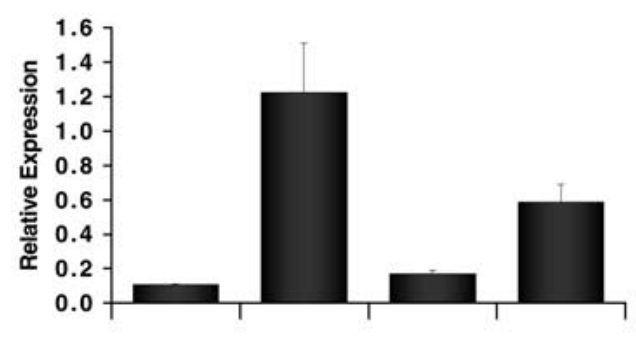

NN CN NC $\propto$

MEG3
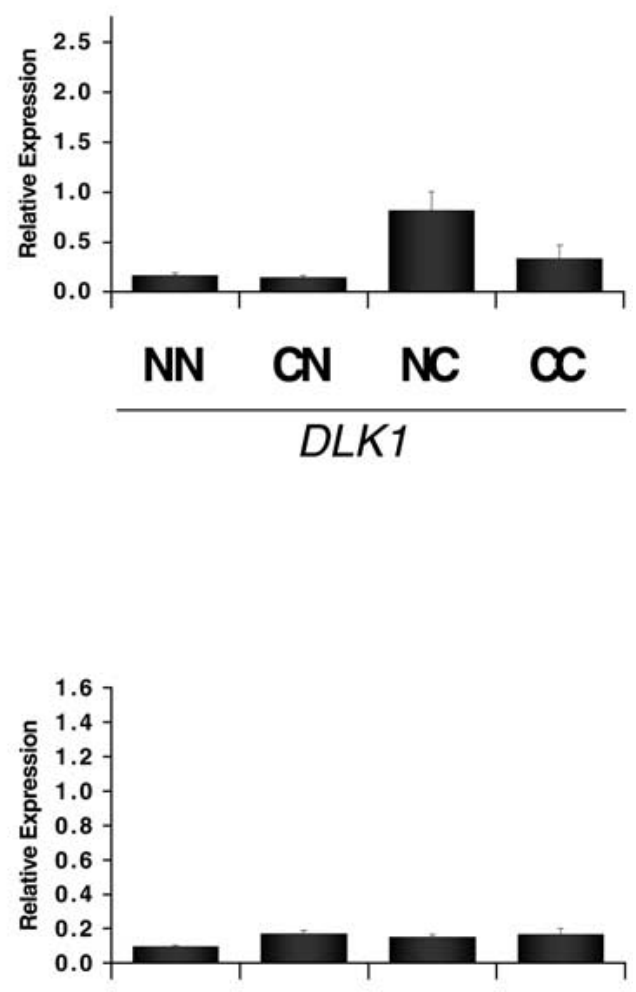

NN CN NC $\propto$

\section{DLK1}

Fig. 4. Quantitative analysis of MEG3 and DLK1 expression in biceps femoris and infraspinatus muscles. Total RNA from biceps femoris muscle was analyzed using RNase protection and normalized to $18 \mathrm{~S}$ rRNA present in each sample to give the relative level of gene expression. (A) Relative expression of MEG3 (left) and DLK1 (right) in adult biceps femoris from $N^{M A T} N^{P A T}(n=2), C^{M A T} N^{P A T}(n=2), N^{M A T} C^{P A T}(n=4)$, and $C^{M A T} C^{P A T}(n=2)$ sheep. (B) Relative expression of $M E G 3$ (left) and DLK1 (right) in adult infraspinatus from $N^{M A T} N^{P A T}(n=2), C^{M A T} N^{P A T}(n=2), N^{M A T} C^{P A T}(n=4)$, and $C^{M A T} C^{P A T}$ $(n=2)$ sheep. Error bars-SEM.

specifically occurs in fast-twitch muscle fibers, i.e., those affected by callipyge.

Little is known about the expression of DLK1 and MEG3 in tissues other than muscle in the callipyge sheep. To investigate whether the patterns of expression for MEG3 and DLK1 in mesodermalderived tissues of the sheep harboring the callipyge mutation extend into tissues from a different embryonic lineage, we performed RNase protection assays in endodermal tissue using the same MEG3 and DLK1 probes (Figs. 5 and 6). In both fetal and adult liver, the relative level of expression of both genes is low compared to muscle (note $Y$-axis), and the overall level of $M E G 3$ is greatly decreased in the adults relative to that found in fetal sheep (7.9-fold averaged across genotypes). Interestingly, in spite of the low RNA levels, MEG3 expression does vary significantly between the $N^{M A T} N^{P A T}$ and $C^{M A T} N^{P A T}$ ( $p=0.031)$ genotypes in adult liver and expression is lower (although not statistically significant) in the $C^{M A T} C^{P A T}$ adults as well (Fig. 5A, Table 1). This pattern of expression, with higher levels of $M E G 3$ in the $N^{M A T} N^{P A T}$ and $N^{M A T} C^{P A T}$ is opposite that observed in the fetal liver and in muscle tissues, indicating independent regulatory mechanisms for MEG3 expression in tissues of mesodermal and endodermal origin.

$D L K 1$ expression is also higher in the fetal liver samples versus adult liver (6.6-fold when averaged across genotypes), and there is a tendency toward higher levels of expression of DLK1 where the callipyge mutation is maternally inherited $\left(C^{M A T} N^{P A T}\right.$ 

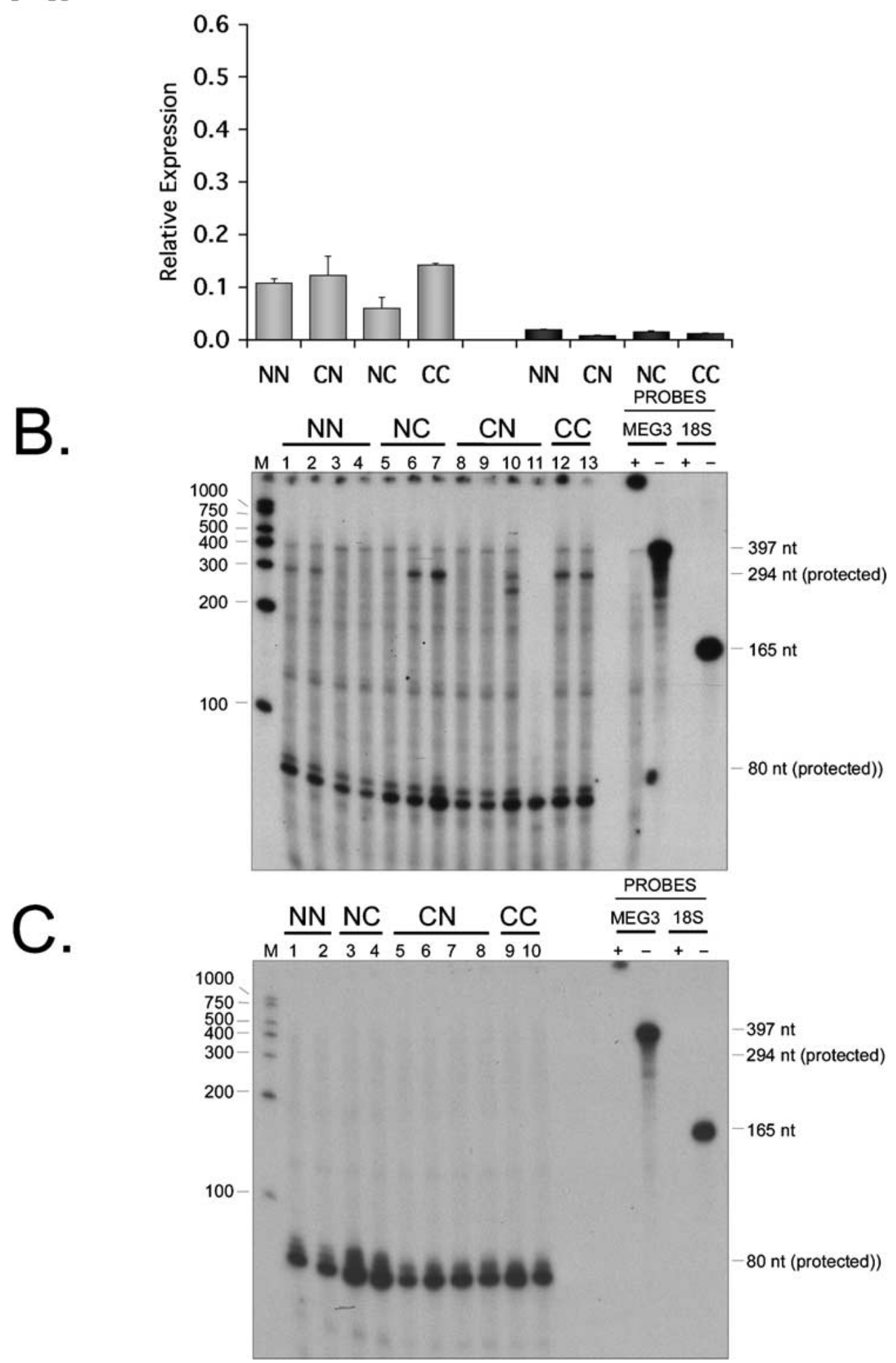

Fig. 5. Quantitative analysis of MEG3 expression in liver of fetal and adult sheep. Total RNA from liver tissue was analyzed using RNase protection and normalized to $18 \mathrm{~S}$ rRNA present in each sample to give the relative level of gene expression. (A) Relative expression of $M E G 3$ in fetal liver (gray bars) from $N^{M A T} N^{P A T}(n=4), C^{M A T} N^{P A T}(n=3), N^{M A T} C^{P A T}$ $(n=4), C^{M A T} C^{P A T}(n=2)$ sheep and adult liver (black bars) from $N^{M A T} N^{P A T}(n=2), C^{M A T} N^{P A T}(n=2), n N^{M A T} C^{P A T}(n=4)$, and $C^{M A T} C^{P A T}(n=2)$ sheep. Error bars-SEM. $(\mathbf{B}, \mathbf{C})$ RNase protection assays used to generate the histogram in panel $(\mathbf{A})$ showing expression of MEG3 in fetal $(\mathbf{B})$ and adult $(\mathbf{C})$ liver. Probes were run alone in the presence $(+)$ or absence $(-)$ of RNase. The MEG3 probe protects 294 nucleotides, while 18S rRNA probe protects a doublet migrating at approximately 80 nucleotides. M-marker lane. 
A.

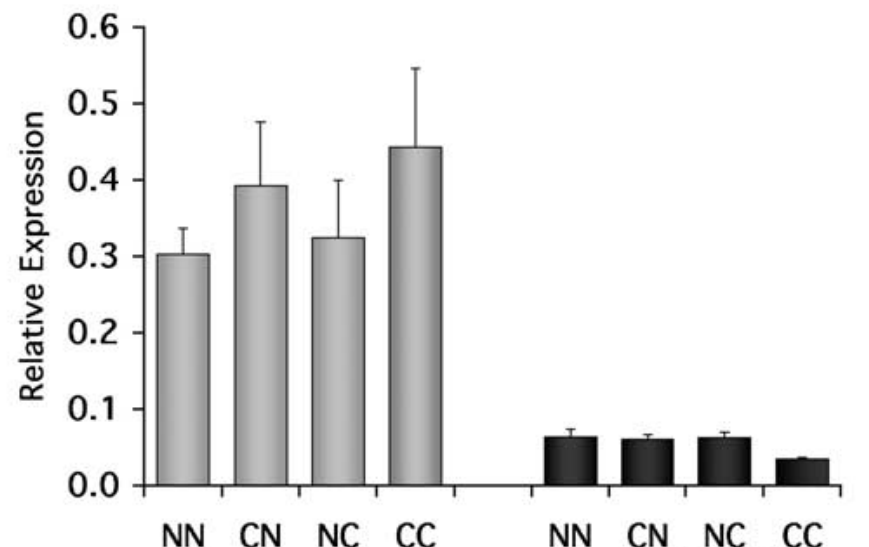

B.

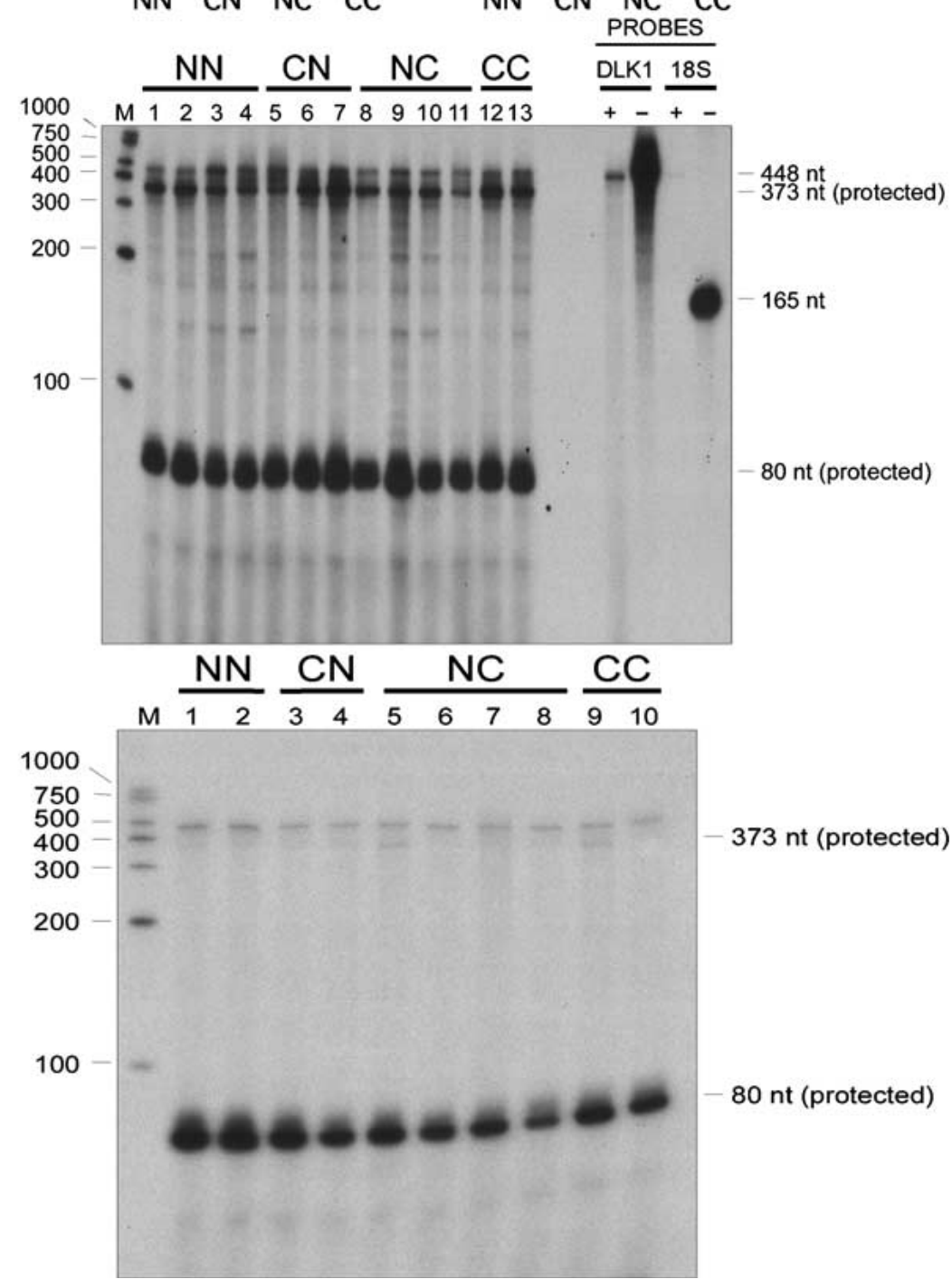

Fig. 6. Quantitative analysis of $D L K 1$ expression in liver of fetal and adult sheep. Total RNA from liver tissue was analyzed using RNase protection and normalized to $18 \mathrm{~S}$ rRNA present in each sample to give the relative level of gene expression. (A) Relative expression of DLK1 in fetal liver (gray bars) from $N^{M A T} N^{P A T}(n=4), C^{M A T} N^{P A T}(n=3), N^{M A T} C^{P A T}$ $(n=4)$, and $C^{M A T} C^{P A T}(n=2)$ sheep and adult liver (black bars) from $N^{M A T} N^{P A T}(n=2), C^{M A T} N^{P A T}(n=2), N^{M A T} C^{P A T}$ $(n=4)$, and $C^{M A T} C^{P A T}(n=2)$, sheep. Error bars-SEM. $(\mathbf{B}, \mathbf{C})$ RNase protection assays used to generate the histogram in panel $(\mathbf{A})$ showing expression of DLK1 in fetal $(\mathbf{B})$ and adult $(\mathbf{C})$ liver. Probes in $(\mathbf{B})$ were run alone in the presence $(+)$ or absence (-) of RNase. The DLK1 probe protects 373 nucleotides, while the $18 \mathrm{~S}$ rRNA probe protects a doublet migrating at approximately 80 mucleotides. M-marker lane. 
unique to the affected $N^{M A T} C^{P A T}$ sheep, these results together suggest that $M E G 3$ is unlikely to be a direct mediator of the muscle hypertrophy phenotype.

Our data clearly show persistent elevation of $D L K 1$ expression in postnatal $N^{M A T} C^{P A T}$ sheep, specifically in callipyge-affected muscles. In another study, DLK1 was found to be elevated in longissimus dorsi muscle of both $N^{M A T} C^{P A T}$ and $C^{M A T} C^{P A T}$ eight-week-old lambs by Northern blot, with decreased levels in the $N^{M A T} N^{P A T}$ and $C^{M A T} N^{P A T}$ sheep (Charlier et al. 2001a). In our study, the average age of the adult sheep was approximately 33 weeks, and at this age the callipyge phenotype is fully evident. The elevated DLK1 in eight-week-old $C^{M A T} C^{P A T}$ lambs suggests that there may be a delay in downregulation of DLK1 expression in the homozygous mutant sheep postnatally, and this delay is apparently not sufficient to give rise to muscular hypertrophy. Combined, these results suggest that the normal attenuation of DLK1 expression takes place perinatally but further work will be required to determine the precise timing of this event.

One mechanism that could account for the normal reduction of $D L K 1$ expression in postnatal sheep is via a trans-regulatory effect (Charlier et al. 2001a; Georges et al. 2003; Charlier 2004). In this scenario, another gene that is reciprocally expressed with $D L K 1$ (e.g., MEG3) may modulate DLK1 expression. Our results indicate that $D L K 1$ expression is normally reduced postnatally, and this reduction appears to be concomitant with an increase in MEG3 expression. Table 1 shows the ratio of the average expression of these two genes for each group of animals in this study. In support of a trans-regulatory role for MEG3, the ratio of DLK1 to MEG3 is high during fetal development in all four genotypes (at a time when the phenotype is not evident), while in adult sheep this ratio remains elevated only in the affected $N^{M A T} N^{P A T}$ animals, and this pattern is observed in the affected muscles. We and others (Charlier et al. 2001a) have noted that expression of genes linked in cis to the callipyge mutation is elevated; as such, the lack of a phenotype in the $C^{M A T} C^{P A T}$ animals may result from the ability of increased MEG3 from the maternal allele to "control" DLK1 that is expressed from the paternal allele. This type of trans effect might occur at the transcriptional or translational level. The results shown in Fig. 3 suggest that this type of regulatory effect may indeed be at the level of transcription since $D L K 1$ transcripts are not elevated in the $C^{M A T} C^{P A T}$ sheep, but more work will be required to test this postulate.

Together with the known biological activities of the DLK1 protein, the combined studies make sheep
DLK1 the strongest candidate to date for mediating the effects of the callipyge mutation. Further support for $D L K 1$ as the callipyge effector gene comes from several lines of evidence. First, pigs also exhibit polar overdominance associated with growth, adiposity, and muscle fiber composition; this is linked to paternal inheritance of DLK1 (Kim et al. 2004). Second, the presence of DLK1 protein was recently shown to be highly specific to fast-twitch muscle fibers in postnatal $N^{M A T} C^{P A T}$ sheep (Davis et al. 2004). Lastly, transgenic mice overexpressing Dlk1 display generalized muscular hypertrophy, linking Dlk1 to the key characteristic of the callipyge phenotype (Davis et al. 2004). The signaling pathways through which DLK1 acts are currently not well understood. Given the role of DLK1 in specifying alternative differentiation fates in vitro, and the increased number of fast-twitch muscle fibers and reduced adiposity in the callipyge sheep, it is possible that sheep DLK1 functions in mesodermal differentiation processes, specifically in the choice between myoblast versus adipocyte cell lineages. Appropriate in vitro and in vivo model systems will be required to investigate this aspect of DLK1 function.

Imprinted genes other than MEG3 and DLK1 are located in the same region and might also contribute to the callipyge phenotype (Charlier et al. 2001b). These include DAT and MEG8, whose human sequence was recently shown to encode snoRNAs (small nucleolar RNAs) (Cavaille et al. 2002). PEG11 is also located in this region and encodes a gag and pol-like polyprotein of unknown function. PEG11 is expressed in sheep skeletal muscle /Charlier et al. 2001a) and is therefore potentially involved in callipyge. PEG11 transcripts are indeed very highly expressed in the affected muscles of postnatal $N^{M A T} C^{P A T}$ sheep compared to the other genotypes, consistent with a specific effect resulting from the presence of the callipyge mutation that correlates with phenotype (Bidwell et al. 2004). However, it is unknown whether PEG11 protein is produced, or how PEG11 may functionally contribute to muscular hypertrophy. In addition, temporal differences in PEG11 expression between fetal and adult sheep remain to be demonstrated. Seitz et al. (2003) established that in mice the previously reported anti-PEG11 (Charlier et al. 2001b) in fact produces microRNAs, and they postulated that these microRNAs may be involved in regulating expression of PEG11. Whether this applies to sheep and whether other microRNAs are present within this imprinted domain that contribute to callipyge muscular hypertrophy is presently unknown. The callipyge mutation is located in a region that is transcribed in sheep to produce CLPG1, an RNA that has the potential to encode protein (Freking et al. 2002). 
However, the open reading frame of this putative protein is not well-conserved across species and, therefore, it is presently unresolved whether CLPG1 has a direct role in the callipyge phenotype. Quantitative expression studies of all of these genes, similar to those described in the current study, are required to answer these questions.

In conclusion, we have provided evidence that the callipyge phenotype correlates with altered temporal expression of the DLK1 gene. Specifically, the appearance of the phenotype coincides with abnormally elevated $D L K 1$ expression in the affected sheep; this is in direct contrast to the downregulation of DLK1 in all of the nonaffected genotypes, including the homozygous $C^{M A T} C^{P A T}$ sheep that are phenotypically normal. Furthermore, substantial $D L K 1$ mRNA transcript elevation is not seen in the nonaffected infraspinatus muscle or in liver of the $N^{M A T} C^{P A T}$ animals; therefore, this variance does not represent a global deregulation of this gene. This evidence, together with the role of $D L K 1$ in cellular differentiation, strongly suggests that $D L K 1$ is an effector of callipyge and that the callipyge mutation is somehow negating the ability to downregulate expression of DLK1 postnatally. Further investigation will be required to determine how the callipyge mutation mechanistically influences the expression of the imprinted genes in the callipyge region.

\section{Acknowledgments}

This work was supported by NIH grants CA94668 to S.K.M., Enterprise Ireland grant IC/2003/20 to C.M.N., and CA25951 and ES08823 to R.L.J.

\section{References}

1. Bidwell CA, Shay TL, Georges M, Beever JE, Berghmans S, et al. (2001) Differential expression of the GTL2 gene within the callipyge region of ovine chromosome 18. Anim Genet 32, 248-256

2. Bidwell CA, Kramer LN, Perkins AC, Hadfield TS, Moody DE, et al. (2004) Expression of PEG11 and PEG11AS transcripts in normal and callipyge sheep. BMC Biol 2, 17

3. Cavaille J, Seitz H, Paulsen M, Fergusor-Smith AC, Bachellerie JP (2002) Identification of tandemly-repeated C/D snoRNA genes at the imprinted human 14q32 domain reminiscent of those at the PraderWilli/Angelman syndrome region. Hum Mol Genet 11, 1527-1538

4. Charlier C (2004) Polar overdominance at the ovine callipyge locus supports trans interaction between the products of reciprocally imprinted genes. In Emerging
Mechanisms of Epigenetic Regulation, Keystone Symposia, Tahoe City, CA, Jan, 21-26, 2004

5. Charlier C, Segers K, Karim L, Shay T, Gyapay G, et al. (2001a) The callipyge mutation enhances the expression of coregulated imprinted genes in cis without affecting their imprinting status. Nat Genet 27, 367369

6. Charlier C, Segers K, Wagenaar D, Karim L, Berghmans $\mathrm{S}$, et al. (2001b) Human-ovine comparative sequencing of a 250-kb imprinted domain encompassing the callipyge (clpg) locus and identification of six imprinted transcripts: DLK1, DAT, GTL2, PEG11, antiPEG11, and MEG8. Genome Res 11, 850-862

7. Cockett NE, Jackson SP, Shay TL, Farnir F, Berghmans $S$, et al. (1996) Polar overdominance at the ovine callipyge locus. Science 273, 236-238

8. Croteau S, Charron MC, Latham KE, Naumova AK (2003) Alternative splicing and imprinting control of the Meg3/Gt12-Dlk1 locus in mouse embryos. Mamm Genome 14, 231-241

9. Davis E, Jensen $\mathrm{CH}$, Schroder HD, Farnir F, Shay-Hadfield T, et al. (2004) Ectopic expression of DLK1 protein in skeletal muscle of padumnal heterozygotes causes the callipyge phenotype. Curr Biol 14, 1858-1862

10. Fahrenkrug SC, Freking BA, Smith TP (1999) Genomic organization and genetic mapping of the bovine PREF1 gene. Biochem Biophys Res Commun 264, 662667

11. Forne T, Oswald J, Dean W, Saam JR, Bailleul B, et al. (1997) Loss of the maternal H19 gene induces changes in Igf2 methylation in both cis and trans. Proc Natl Acad Sci USA 94, 10243-10248

12. Freking BA, Keele JW, Beattie CW, Kappes SM, Smith TP, et al. (1998a) Evaluation of the ovine callipyge locus: I. Relative chromosomal position and gene action. J Anim Sci 76, 2062-2071

13. Freking BA, Keele JW, Nielsen MK, Leymaster KA (1998b) Evaluation of the ovine callipyge locus: II. Genotypic effects on growth, slaughter, and carcass traits. J Anim Sci 76, 2549-2559

14. Freking BA, Murphy SK, Wylie AA, Rhodes SI, Keele JW, et al. (2002) Identification of the single base change causing the callipyge muscle hypertrophy phenotype, the only known example of polar overdominance in mammals. Genome Res 12, 1496-1506

15. Georges M, Charlier C, Cockett N (2003) The callipyge locus: evidence for the trans interaction of reciprocally imprinted genes. Trends Genet 19, 248-252

16. Kim K, Kim J, Dekkers JCM, Rothschild MF (2004) Polar overdominant inheritance of a $D L K 1$ polymorphism is associated with growth and fatness in pigs. Mamm Genome 15, 552-559

17. Koohmaraie M, Shackelford SD, Wheeler TL, Lonergan SM, Doumit ME (1995) A muscle hypertrophy condition in lamb (callipyge): characterization of effects on muscle growth and meat quality traits. J Anim Sci 73, 3596-3607

18. Laborda J (2000) The role of the epidermal growth factor-like protein dlk in cell differentiation. Histol Histopathol 15, 119-129 
19. Laborda J, Sausville EA, Hoffman T, Notario V (1993) Dlk, a putative mammalian homeotic gene differentially expressed in small cell lung carcinoma and neuroendocrine tumor cell line. J Biol Chem 268, 3817-3820

20. Li YM, Franklin G, Cui HM, Svensson K, He XB, et al. (1998) The $H 19$ transcript is associated with polysomes and may regulate IGF2 expression in trans. J Biol Chem 273, 28247-28252

21. Moon YS, Smas CM, Lee K, Villena JA, Kim KH, et al. (2002) Mice lacking paternally expressed Pref-1/Dlk1 display growth retardation and accelerated adiposity. Mol Cell Biol 22, 5585-5592

22. Paulsen M, Takada S, Youngson NA, Benchaib M, Charlier C, et al. (2001) Comparative sequence analysis of the imprinted Dlk1-Gtl2 locus in three mammalian species reveals highly conserved genomic elements and refines comparison with the Igf2-H19 region. Genome Res 11, 2085-2094

23. Runge S, Nielsen FC, Nielsen J, Lykke-Andersen J, Wewer UM, et al. (2000) H19 RNA binds four molecules of insulin-like growth factor II mRNA-binding protein. J Biol Chem 275, 29562-29569

24. Seitz H, Youngson N, Lin SP, Dalbert S, Paulsen M, et al. (2003) Imprinted microRNA genes transcribed antisense to a reciprocally imprinted retrotransposonlike gene. Nat Genet 34, 261-262
25. Smas CM, Sul HS (1993) Pref-1, a protein containing EGF-like repeats, inhibits adipocyte differentiation. Cell 73, 725-734

26. Smit M, Segers K, Carrascosa LG, Shay T, Baraldi F, et al. (2003) Mosaicism of solid gold supports the causality of a noncoding A-to-G transition in the determinism of the callipyge phenotype. Genetics 163, 453-456

27. Takada S, Paulsen $M$, Tevendale $M$, Tsai CE, Kelsey G, et al. (2002) Epigenetic analysis of the Dlk1-Gt12 imprinted domain on mouse chromosome 12: implications for imprinting control from comparison with Igf2-H19. Hum Mol Genet 11, 77-86

28. Van Limpt V, Chan A, Caron H, Sluis PV, Boon K, et al. (2000) SAGE analysis of neuroblastoma reveals a high expression of the human homologue of the Drosophila Delta gene. Med Pediatr Oncol 35, 554-558

29. Van Limpt VA, Chan AJ, Van Sluis PG, Caron HN, Van Noesel CJ, et al. (2003) High delta-like 1 expression in a subset of neuroblastoma cell lines corresponds to a differentiated chromaffin cell type. Int J Cancer 105, 61-69

30. Wylie AA, Murphy SK, Orton TC, Jirtle RL (2000) Novel imprinted DLK1/GTL2 domain on human chromosome 14 contains motifs that mimic those implicated in IGF2/H19 regulation. Genome Res 10, $1711-1718$ 